\title{
BMJ Open Transitioning from sickness absence to disability pension - the impact of poor health behaviours: a prospective Swedish twin cohort study
}

\author{
Björg Helgadóttir, ${ }^{1}$ Lisa Mather, ${ }^{1}$ Jurgita Narusyte, ${ }^{1}$ Annina Ropponen (D) , ${ }^{1,2}$ \\ Victoria Blom, ${ }^{1,3}$ Pia Svedberg (i) ${ }^{1}$
}

To cite: Helgadóttir B, Mather L, Narusyte J, et al. Transitioning from sickness absence to disability pension - the impact of poor health behaviours: a prospective Swedish twin cohort study. BMJ Open 2019;9:e031889. doi:10.1136/ bmjopen-2019-031889

- Prepublication history for this paper is available online. To view these files, please visit the journal online (http://dx.doi. org/10.1136/bmjopen-2019031889).

Received 23 May 2019 Revised 07 October 2019 Accepted 18 0ctober 2019
Check for updates

(C) Author(s) (or their employer(s)) 2019. Re-use permitted under CC BY-NC. No commercial re-use. See rights and permissions. Published by BMJ.

${ }^{1}$ Division of Insurance Medicine, Clinical Neuroscience,

Karolinska Institutet, Stockholm, Sweden

${ }^{2}$ Finnish Institute of Occupational Health, Tyoterveyslaitos,

Helsinki, Finland

${ }^{3}$ Swedish School of Sport and Health Sciences, Stockholm, Sweden

Correspondence to PhD Pia Svedberg; pia.svedberg@ki.se

\section{ABSTRACT}

Objectives To investigate the association between three poor health behaviours (current smoker, high consumption of alcohol and low physical activity levels) and the transition to disability pension (DP) among individuals who have recently been sickness absent. Furthermore, we aimed to explore whether having multiple poor health behaviours increased the risk of transitioning from sickness absence (SA) to DP.

Design Prospective twin cohort study.

Setting Sweden.

Participants Twins aged 20-46 who had participated in a survey and been on SA (>14 days) in the year preceding baseline (date of answering the questionnaire).

Main outcome measure Incident DP during the follow-up which ended on 31 December 2012 (mean 5.2 years). A national register with full coverage provided data on DP. Results The Cox proportional-hazards regression analyses showed that current smokers had a higher risk of transitioning from SA to DP compared with never smokers (HR 1.76; 95\% $\mathrm{Cl} 1.08$ to 2.84). Alcohol use and lack of physical activity as well as poor health behaviour sum score showed no significant associations.

Conclusions Being a current smoker influences the transition from SA to DP. Although non-significant, there were indications that more physical activity and fewer poor health behaviours could reduce the risk of exiting the labour market through DP. Improving health behaviours among people on SA could be a valuable tool for preventing the transition to DP.

\section{INTRODUCTION}

The burden of sickness absence (SA) in Sweden and many other Western societies is a growing public health problem ${ }^{12}$ and the costs to society are high. ${ }^{3}$ Although SA is meant to give the individual time to heal, there are also negative side effects such as lower income, as well as social and psychological consequences in terms of feeling isolated and powerless. ${ }^{45}$ For most people, SA is a temporary situation, but a small percentage do not re-join the labour force but instead
Strengths and limitations of this study

- This study used a unique combination of national register and survey data.

- There was complete coverage of sickness absence (>14 days) and disability pension.

- Clear temporal relationship: sickness absence occurred before the collection of survey data (health behaviours)

- The relatively small sample was a limitation leading to insufficient power for discordant twin analyses

- The poor health behaviours, especially alcohol use, had internal missing which consequently lead to low precision.

permanently exit the labour market through disability pension (DP). ${ }^{6}$

Previous investigations of risk factors for transitioning from SA to DP show that higher age, being a woman, musculoskeletal and mental disorders, and lower socioeconomic status are important predictors. ${ }^{7-10}$ However, less is known about the impact of poor health behaviours, such as smoking, alcohol consumption and physical inactivity, although indications exist that these behaviours increase the risk of SA and DP. ${ }^{11}$ Smoking is a major risk factor for ill health ${ }^{12}$ and is associated with a higher risk of $\mathrm{SA}^{1314}$ and DP. ${ }^{15}$ Alcohol consumption is known to be associated with both higher frequency of SA and longer SA duration, ${ }^{15} 16$ though one study showed that alcohol intake tended to decrease rather than increase after SA. ${ }^{17}$ Being physically inactive during leisure time has been shown to be linked with a higher risk of $\mathrm{SA}^{1819}$ and $\mathrm{DP}^{2021} \mathrm{~A}$ review of intervention studies has shown that physical activity, such as resistance training and moderate to vigorous physical activity can reduce $\mathrm{SA}$, though the evidence is limited. ${ }^{22}$ However, not all physical activity is protective 
as occupational physical activity can lead to a higher risk of $\mathrm{SA},{ }^{23}$ indeed the more exposed a person is to lifting and kneeling the more likely they are to become SA. ${ }^{24}$

Poor health behaviours also tend to cluster. ${ }^{25}$ Smoking (nicotine dependence) has been shown to be associated with alcohol abuse disorders, ${ }^{26}$ and a study with an exceptionally long follow-up of 35 years showed that being a smoker can increase the risk of remaining or becoming physically inactive. ${ }^{27}$ Furthermore, having multiple poor health behaviours increase the risk of DP due to musculoskeletal disorders, though only after adjustment for familial factors (genes and shared environment). The results for DP due to other diagnoses (mental and circulatory system diagnoses) were less clear and interaction analyses showed a complex relationship between the different health behaviours and DP. ${ }^{28}$ Also, a couple of reviews suggest that interventions that target multiple health behaviours at once might be more effective than interventions for a single behaviour, ${ }^{29}{ }^{30}$ although not related to SA or DP specifically. However, further investigations are needed, more specifically into whether poor health behaviours influence the transition from SA to DP.

The aim of this study was to investigate the associations between three poor health behaviours (smoking, alcohol consumption and low physical activity) and the transition to DP among people who have recently been sickness absent. The poor health behaviours were explored as separate risk factors but also as a sum score of poor health behaviours.

\section{METHODS}

This was a prospective twin cohort study based on data from the Swedish Twin project Of Disability pension and Sickness absence (STODS) which includes survey and national register data. Twins born between 1959 and 1985 were invited to answer the web-based questionnaire Study of Twin Adults: Genes and Environment (STAGE) in 2004-2006 conducted by the Swedish Twin Registry (STR). ${ }^{31}$ In total, 25496 twins answered the STAGE survey and baseline was set on the date that each participant answered the questionnaire.

\section{Participants}

We included those that had been sickness absent $(>14$ days) at least once during the year preceding baseline $(n=2054)$. We excluded those who at baseline had emigrated $(n=1)$ or were on DP $(n=62)$. The final sample included in total 1991 twins (see figure 1). By using this design, we ensured that the information on health behaviours was collected after the participants had been sickness absent.

\section{Registers used}

Data were obtained from the following national registers: (1) MiDAS held by the National Social Insurance Agency contains information on SA and DP including diagnosis, duration and grade. (2) LISA held by Statistics Sweden

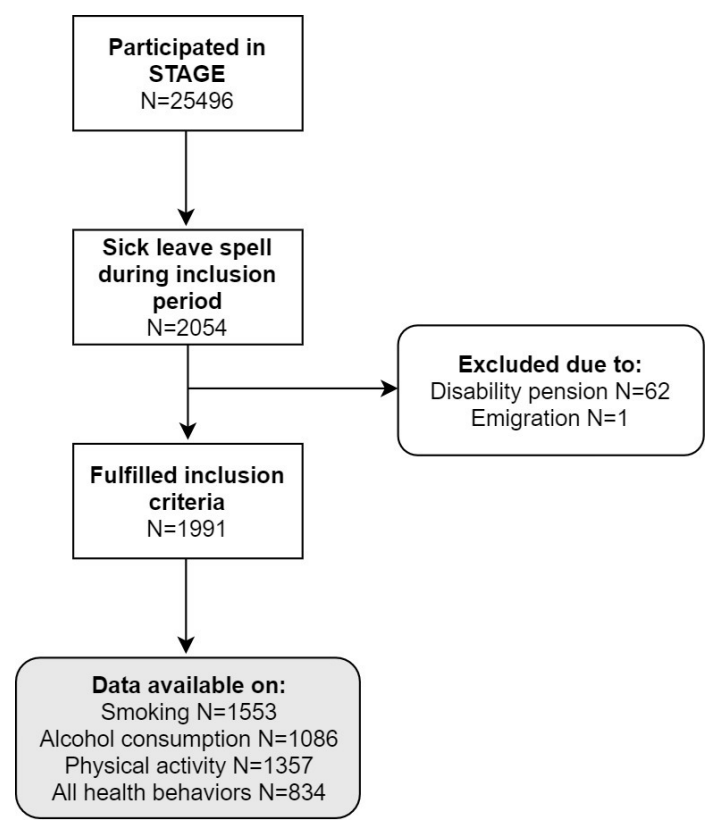

Figure 1 Flow chart of the study participants.

contains information on old age pension, work status and education. ${ }^{32}$ (3) Cause of death register held by the National Board of Health and Welfare contains information on the date of death. The data from these registers and questionnaire data were linked using the Swedish 10-digit personal identification number.

\section{Swedish social security system}

All individuals in Sweden above the age of 16, with an income from work or unemployment benefits, can receive sick leave benefits paid by the Social Insurance Agency when disease or injury has caused reduced work capacity. Employees receive sick pay from their employers during the first 14 days after a qualifying day without benefits (self-employed usually have more qualifying days). After this period, the employees receive up to $80 \%$ of lost income from the Social Insurance Agency. Unemployed individuals have one qualifying day and receive sick pay from the Social Insurance Agency from the second day. Duration of SA was calculated in net days, that is, taking into account the occurrence of part-time SA.

A national social security DP scheme covers all citizens in Sweden, and to be eligible for disability pension benefits, a medically confirmed disorder or injury is required that permanently reduces work capacity by at least $25 \%$. The Social Insurance Agency can grant a DP that covers about $65 \%$ of lost income based on a thorough assessment of the level of work incapacity.

\section{Twin data}

Data from twins are completely comparable with that of the general population but have the advantage of allowing for adjustment and exploration of familial factors, that is, shared environment and genetics. However, in this study, we did not have enough power to use the twin design. However, all models were corrected for the 
non-independence of the twin pairs and adjustments were made for zygosity.

\section{Health behaviors}

The questions: "Have you ever smoked or used snuff?", "Do you currently smoke cigarettes?" and "Do you currently smoke cigarettes occasionally or at parties" were used to categorise the respondents into never, former or current smoker (of cigarettes) with never smoker set as the reference group. Note that those that used snuff (oral tobacco) were not classified as smokers. Detailed questions of alcohol consumption, which included type, amount and frequency, were summarised into $\leq 100 \mathrm{~g}$ per week or $>100 \mathrm{~g}$ per week, according to recently suggested cut-offs, ${ }^{33}$ and the $\leq 100 \mathrm{~g}$ per week was used as a reference. Participants were asked to rate their current total physical activity level including occupational physical activity, housework, transportation, exercise and other leisure-time physical activity. ${ }^{34}$ Physical activity was rated on a scale from 1 to 10 , where $1=$ verylow (mainly sedentary), $5=$ moderate physical activity (a few walks per week) and 10=very high (sports/jogging several times a week). We collapsed the scale into 1-4 (sedentary/low), 5-6 (moderate), 7-8 (high) and 9-10 (vigorous).

Furthermore, we made a sum score of poor health behaviours in which we added up the three health behaviours to capture the total number. For this sum score, the physical activity was dichotomised into 1-6 and 7-10. In addition, the categories of former and never smoker was collapsed. The total health behaviour score could, therefore, take values from zero poor health behaviours to three poor health behaviours. ${ }^{28}$

\section{Disability pension}

The participants were followed up for all-cause DP from baseline to 31 December 2012. Participants were censored for death and emigration. Emigration date was set at the beginning of the first of the 2 years that were missing in the LISA register.

\section{Covariates}

Other covariates from the questionnaire were marital status (married/cohabiting or other), height in metres (m) and weight in kilograms $(\mathrm{kg})$ which were used to calculate the body mass index (BMI, $\mathrm{kg} / \mathrm{m}^{2}$ ). Sex and age (at the time of the STAGE interview) came from the STR. Information on education (elementary, secondary or higher education), having children under 18 living at home (yes/no) and type of living environment (urban or semi-urban/rural) were obtained from the LISA register.

\section{Statistical analyses}

The association between each health behaviour and DP was estimated using Cox proportional-hazards regression analyses. The results were presented as HRs with $95 \%$ CIs. The unit of the follow-up time was days and analyses were conducted with censoring for DP (outcome), emigration, date of death or end of study, whichever came first. The proportional-hazard assumption was explored via global test and graphs. The global test for physical activity was significant $(\mathrm{p}=0.048)$. We tested the fit of the model with and without time-varying covariates, but the fit was very similar, although slightly better without, based on Akaike Information Criterion, hence those results are presented. The crude model included each health behaviour and the poor health behaviour sum score, respectively. The following models were then adjusted for age, zygosity (model 1), education, children under 18 living at home, marital status and type of environment (model 2); each model was adjusted for what was included in the previous models. In models 1 and 2, all HRs were based on stratification by sex to allow men and women to have their own baseline hazards. Each model included only those participants who had complete data on each health behaviour and confounders (see figure 1). Furthermore, we did sensitivity analyses where we adjusted for BMI and the other two health behaviours, respectively, as well as using another method of classifying alcohol use, that is, 9 standard drinks for women and 14 drinks for men per week. ${ }^{35}$ These yielded similar results as other models hence we chose not to report the results (data not shown).

In all the models, the SEs were adjusted for pair identity to consider the within-pair correlations. Analyses were conducted using STATA IC V.12.1 and the significance level was set at $\alpha=0.05$.

Sensitivity analyses where we first added BMI and then the other two health behaviours to the models yielded similar results. Furthermore, we explored using other cutoffs for alcohol including 9 standard drinks for women and 14 drinks for men per week. The results were similar though the estimates were slightly stronger (data not shown).

\section{Patient and public involvement}

The study participants or the general public were not involved in decisions about the research question, the design of the study, the outcomes, the conduct of the study, the drafting of the paper nor in the dissemination of the study results.

\section{RESULTS}

Most participants (80.4\%) had been on SA for less than 90 net days before the baseline, while $8.3 \%$ had been on SA for 91-180 net days and $11.3 \%$ had 181 net days or longer SA. Of the 1991 individuals on SA at the baseline, $124(6.2 \%)$ went on DP during follow-up. The incidence rate per 100 person years was 35.4 (95\% CI 29.7 to 42.2). The average duration of follow-up was 5.2 years. The majority were women $(72.4 \%)$ and the mean age was 34.7 years (SD 6.7). Around one-fifth were current smokers, $14.7 \%$ drank $\geq 100 \mathrm{~g}$ of alcohol per week and $14.8 \%$ were categorised as being sedentary or having low physical activity (table 1). Being a current smoker was associated with a higher risk of moving from SA to DP compared with a never smoker (HR 1.76; 95\% CI 1.08 to 2.84). No statistically significant associations were found for the 
Table 1 Baseline characteristics of the 1991 study participants with a sick leave spell during the inclusion period

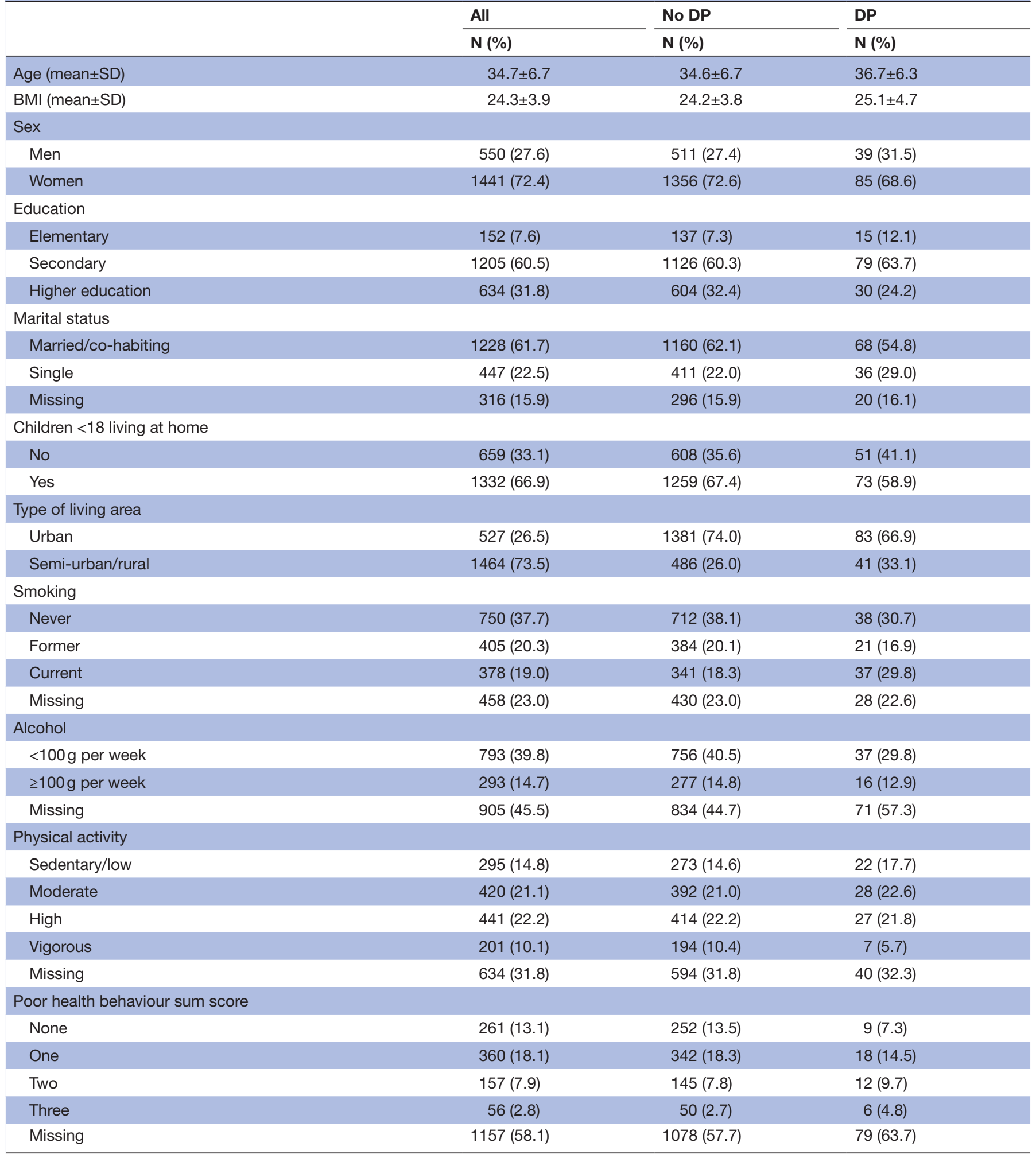

Only questionnaire data have missing values as the registers have complete coverage.

$\mathrm{BMI}$, body mass index; DP, disability pension.

other health behaviours even though estimates were indicating that vigorous physical activity seems protective of DP compared with sedentary/low activity. For the poor health behaviour sum score, no statistically significant associations were found in the fully adjusted model. However, having multiple poor health behaviours showed a tendency for a higher risk of DP with an increased number of poor behaviours (HR three poor behaviours: 
Table 2 Results from the crude and adjusted Cox regression models of the associations between poor health behaviours and disability pension (DP) among participants with a sick leave spell during inclusion period, HRs with $95 \%$ Cls

\begin{tabular}{|c|c|c|c|}
\hline & Crude model & Model 1 & Model 2 \\
\hline & HR (95\% Cl) & HR (95\% Cl) & HR (95\% Cl) \\
\hline \multicolumn{4}{|l|}{ Smoking $(n=1533)$} \\
\hline Former & 1.02 (0.60 to 1.73$)$ & $1.04(0.60$ to 1.78$)$ & 0.99 (0.57 to 1.70$)$ \\
\hline Current & $2.00(1.27$ to 3.14$)$ & $2.11(1.33$ to 3.35$)$ & 1.76 (1.08 to 2.84$)$ \\
\hline \multicolumn{4}{|l|}{ Alcohol $(n=1086)$} \\
\hline$<100 \mathrm{~g}$ per week & Ref & Ref & Ref \\
\hline$\geq 100 \mathrm{~g}$ per week & 1.21 (0.67 to 2.18$)$ & 1.12 (0.62 to 2.05$)$ & 0.96 (0.54 to 1.74$)$ \\
\hline \multicolumn{4}{|c|}{ Physical activity $(n=1357)$} \\
\hline Sedentary/low & Ref & Ref & Ref \\
\hline Moderate & 0.92 (0.52 to 1.62$)$ & 0.91 (0.52 to 1.60$)$ & 0.94 (0.53 to 1.65$)$ \\
\hline \multicolumn{4}{|c|}{ Poor health behaviour sum score $(n=834)$} \\
\hline None & Ref & Ref & Ref \\
\hline One & 1.52 (0.69 to 3.35$)$ & 1.48 (0.66 to 3.29$)$ & 1.55 (0.71 to 3.36$)$ \\
\hline Two & 2.36 (1.00 to 5.58$)$ & 2.36 (0.95 to 5.81$)$ & 2.23 (0.92 to 5.42$)$ \\
\hline Three & 3.33 (1.19 to 9.33$)$ & 3.18 (1.13 to 8.96$)$ & 2.57 (0.92 to 7.19$)$ \\
\hline
\end{tabular}

Model 1: Adjusted for age, zygosity as well as separate baseline hazard for men and women.

Model 2: Same as model 1 plus adjusted for education, children at home, marital status, type of environment.

The $\mathrm{n}$ applies to the crude models.

Statistically significant HR in bold.

$2.57 ; 95 \%$ CI 0.92 to 7.19$)$ ) compared with no poor health behaviours. Results are presented in table 2.

\section{DISCUSSION}

This study used both questionnaire and register data to investigate the influence of poor health behaviours on the transitions from SA to DP. Poor health behaviours, including smoking, high alcohol consumption and low levels of physical activity, are known to affect the risk of diseases and are therefore likely to affect whether people transition back to the labour market after a sick leave spell or permanently exit from labour market, that is, DP. In this study, we found that smoking was a relatively strong risk factor for the transition to DP after SA spells.

Being physically inactive is a risk factor for SA and $\mathrm{DP}^{1821}$ and is well established as a risk factor for poor physical and mental health. ${ }^{36}$ Although no significant effects were seen in this study for physical activity, there was a tendency towards decreased risk of transitioning to DP with higher levels of physical activity. This finding is in line with a previous meta-analysis which indicate that lack of physical activity is associated with DP. ${ }^{38}$ This result is also in line with previous studies that have found a doseresponse between physical activity and $\mathrm{DP}^{21}$ and $\mathrm{SA},{ }^{18}$ and mortality. ${ }^{39}$ However, a larger study population is needed to confirm our results. Nevertheless, low levels of physical activity seem to be a risk factor for SA and DP, respectively, and also seem to be of importance for the prognosis of DP among those with a history of SA.

High levels of alcohol consumption had no effect on the risk of transitioning from SA to DP. However, there could still be a risk associated with high consumption of alcohol that was not captured by this study. Being male and a heavy drinker has previously shown to be associated with a higher risk of $\mathrm{SA}^{40}$ and for both women and men a higher risk for $\mathrm{DP} .^{41}$ In addition, a natural experiment showed that those that had more access to alcohol in adolescents were more likely to go on DP in adulthood. ${ }^{42}$

Poor health behaviours also tend to cluster. ${ }^{43}$ In this study, we found that having multiple poor health behaviours showed a tendency for a higher risk of DP with an increased number of poor behaviours though the results were not significant in the fully adjusted model. A previous study on the additive effects of having multiple poor health behaviours on the risk of DP showed a complex interrelationship between health behaviours and different DP diagnoses. ${ }^{28}$ In addition, earlier systematic reviews on the clustering of poor health behaviours suggest that interventions that target multiple health behaviours at once might be more effective than interventions for a single behaviour. ${ }^{29}{ }^{30}$ This leads to implications for clinical practice as the individuals' behaviour pattern 
needs to be a whole to prevent them from transitioning from SA to DP.

We attempted to run twin models, but these are based on twin pairs that are discordant on the exposure to be informative and the number of twin pairs in our data was not enough to get any meaningful results from these analyses. We have previously shown that familial factors, that is, genes and shared environment primarily while growing up, can play a role when studying associations between various exposures and $\mathrm{SA}^{44}$ and DP. ${ }^{45}$ Thus, a discordant twin pair analysis would have added a dimension beyond the analyses based on singleton populations. Moreover, in some previous studies regarding associations between health behaviours and SA or DP, either BMI or obesity was included. ${ }^{11}$ In this study, we were not focusing on BMI and the potential influence of BMI on the transition from SA to DP, but we considered body weight as a potential confounder. Sensitivity analyses included adjustment for BMI and the other health behaviours including smoking, physical activity and so on, but the adjustments had no effects on the estimates.

\section{Strengths and limitations}

One of the main strengths of the study is the combination of survey and register data. Register data provided complete coverage of SA longer than 14 days as well as coverage of DP with an average follow-up of 5.2 years. However, register data do not include data on health behaviours which is where survey data is an advantage. In this study, it was important to know exactly which came first, the health behaviour or SA, and as there was precise information on when the survey data were collected and when the SA spell began, we could explore how health behaviour after SA affects the transition to DP.

However, the design of the study was not without drawbacks. Since we restricted to those that had been on SA in the year preceding the survey, the sample was small, leading to insufficient statistical power for the discordant twin analyses. The missing was especially problematic on the health behaviour sum score. Analyses on those that were missing on the poor health behaviour sum score showed that they were younger ( 34.1 vs 35.6 years $\mathrm{p}=0.00)$ and more likely to be women $(\mathrm{p}=0.04)$. But no statistically significant differences were found regarding education, marital status and DP. As for the separate health behaviours, alcohol consumption had the highest proportion of missing. Those that had missing information regarding alcohol were more likely to transition to DP $(\mathrm{p}=0.006)$; in other respects, the pattern was similar to the missing on the poor health behaviour sum score. Hence, we may have underestimated the true effect and it is therefore suggested that the analyses should be repeated in a larger sample if available. In addition, a larger sample would have allowed us to explore different diagnoses of SA and DP which might have been very informative as the impact of health behaviour could be different for different underlying diagnoses for SA/ DP. Moreover, although the health behaviour data were collected after the SA episodes, it is likely that several of the health behaviours started much earlier in life for many participants. Thus, earlier and long-lasting poor health behaviours could be influencing both the risk of SA and DP. In this study, we have no information regarding when the behaviours started, hence we could not take this into account.

Our sample was rather homogeneous regarding alcohol consumption as demonstrated by the fact that no individuals were classified as abstainers, who have previously been shown to have a higher risk of SA. ${ }^{40}$ We also explored different ways of defining high alcohol consumption taking sex into account. In such an approach, 9 standard alcoholic drinks was the cut-off for women whereas 14 drinks for men per week was used, but we found very similar results to those presented, though with slightly stronger effects. The questions on alcohol had a large proportion of internal missing which contributed to the low power for this measure. Questions on alcohol consumption are often considered as rather sensitive in nature which could have contributed to the high number of missing responses. In addition, social desirability bias might have influenced the respondents towards underreporting their consumption which might have led to misclassification of alcohol consumption. ${ }^{46}$ The questions on alcohol were comprehensive though it might also have contributed to low response as the questions might have been perceived as overwhelming.

We chose a priori to define low physical activity as scoring 6 or lower on the physical activity question. This might be a rather high cut-off, as in the question a score of 5-6 was defined as being moderately active. However, people have been shown to overestimate their physical activity level in questionnaire data when compared with data from objective measurements, ${ }^{47}{ }^{48}$ possibly due to recall or social desirability bias. ${ }^{46}{ }^{49}$ It is therefore not unreasonable to expect that our measurement of physical activity would have inflated the true level of physical activity in our study population. Furthermore, the moderate group is very close to the estimate of none/low physical activity in the survival analyses of the separate health behaviours, while a larger jump was seen for the high physical activity category.

\section{CONCLUSIONS}

The transition from SA to DP was influenced by smoking, but physical activity also played a role. In addition, there might be additive effects of having multiple poor health behaviours, though further exploration is needed on larger samples. Supporting people on SA to improve their health behaviour might be a promising approach to prevent exit from the labour market through DP.

Twitter Annina Ropponen @AnninaRop

Acknowledgements We acknowledge the Swedish Twin Registry for access to data. 
Contributors PS originated the idea. $\mathrm{BH}$ analysed the data in consultation with LM, PS, JN, VB and AR. BH wrote the first and subsequent drafts of the manuscript with important intellectual input from all the coauthors. All authors contributed in designing the study and to the interpretation of the results as well as to the writing and approval of the final version of the article.

Funding This study was financially supported by AFA Insurance (160138). The Swedish Twin project Of Disability pension and Sickness absence (STODS) is supported by the Swedish Research Council under grant no. 2017-00624. The Swedish Twin Registry is managed by Karolinska Institutet and receives funding through the Swedish Research Council under grant no. 2017-00641. The Study of Twin Adults: Genes and Environment (STAGE) was supported by the National Institute of Health, USA (grants DK 066134 and CA 085739).

Disclaimer The study participants or the general public were not involved in any part of this study.

Competing interests None declared.

Patient consent for publication Not required

Ethics approval The study population was identified by linking several nationwide registers. In Sweden, ethical approval is always required when using sensitive register data from authorities. The ethical vetting was performed and approved by the Regional Ethical Review Board of Stockholm, Sweden (Dnr: 2007/52431, Dnr: 2010/1346-32/5 and Dnr: 2014/311-32), according to the Swedish Ethical Review Act and after that also by each of the different authorities/data keepers (Statistics Sweden and the National Social Insurance Agency) according to the Public Access to Information and Secrecy Act, the Personal Data Act and the Administrative Procedure Act. The Regional Ethical Review Board can waive the requirement to consult in these type of large register studies, and for this project stated that the consent to participate was not applicable. However, informed consent was obtained for participants in STAGE.

Provenance and peer review Not commissioned; externally peer reviewed.

Data availability statement The data cannot be made publically available. According to the General Data Protection Regulation, the Swedish law SFS 2018:218, the Swedish Data Protection Act, the Swedish Ethical Review Act, and the Public Access to Information and Secrecy Act, these type of sensitive data can only be made available after legal review, for researchers who meet the criteria for access to this type of sensitive and confidential data. Readers may contact Associate Professor Pia Svedberg (pia.svedberg@ki.se) regarding the data.

Open access This is an open access article distributed in accordance with the Creative Commons Attribution Non Commercial (CC BY-NC 4.0) license, which permits others to distribute, remix, adapt, build upon this work non-commercially, and license their derivative works on different terms, provided the original work is properly cited, appropriate credit is given, any changes made indicated, and the use is non-commercial. See: http://creativecommons.org/licenses/by-nc/4.0/.

\section{ORCID iDs}

Annina Ropponen http://orcid.org/0000-0003-3031-5823

Pia Svedberg http://orcid.org/0000-0001-7952-3418

\section{REFERENCES}

1 Swedish Social Insurance Agency. Health and social care have the highest number of new sick leave cases in Sweden. [In Swedish: Vàrd och omsorg har flest nya sjukfall i Sverige]. in Korta analyser, 2015.

2 Gimeno Det al. Distribution of sickness absence in the European Union countries. Occup Environ Med 2004;61:867-9.

3 de Vroome EMM, Uegaki K, van der Ploeg CPB, et al. Burden of sickness absence due to chronic disease in the Dutch workforce from 2007 to 2011. J Occup Rehabil 2015;25:675-84.

4 Vingard E, Alexanderson K, Norlund A. Swedish Council on technology assessment in health care (SBU). Chapter 9. consequences of being on sick leave. Scand J Public Health Supp 2004;63:207-15.

5 Lännerström L, Wallman T, Holmström IK. Losing independence-the lived experience of being long-term sick-listed. BMC Public Health 2013;13:745.

6 Kivimäki M, Forma P, Wikström J, et al. Sickness absence as a risk marker of future disability pension: the 10-town study. J Epidemiol Community Health 2004;58:710-1.

7 Gjesdal S, Bratberg E, Maeland JG. Musculoskeletal impairments in the Norwegian working population: the prognostic role of diagnoses and socioeconomic status: a prospective study of sickness absence and transition to disability pension. Spine 2009;34:1519-25.

8 Gjesdal S, Bratberg E, Mæland JG. Gender differences in disability after sickness absence with musculoskeletal disorders: fiveyear prospective study of 37,942 women and 26,307 men. BMC Musculoskelet Disord 2011;12.

9 Bratberg E, Gjesdal S, Maeland JG. Sickness absence with psychiatric diagnoses: individual and contextual predictors of permanent disability. Health Place 2009;15:308-14.

10 Dorner TE, Alexanderson K, Svedberg P, et al. Sickness absence due to back pain or depressive episode and the risk of all-cause and diagnosis-specific disability pension: a Swedish cohort study of 4,823,069 individuals. Eur J Pain 2015;19:1308-20.

11 Virtanen M, Ervasti J, Head J, et al. Lifestyle factors and risk of sickness absence from work: a multicohort study. Lancet Public Health 2018;3:e545-54.

12 Lim SS, Vos T, Flaxman AD, et al. A comparative risk assessment of burden of disease and injury attributable to 67 risk factors and risk factor clusters in 21 regions, 1990-2010: a systematic analysis for the Global Burden of Disease Study 2010. The Lancet 2012;380:2224-60.

13 Alavinia SM, van den Berg TIJ, van Duivenbooden C, et al. Impact of work-related factors, lifestyle, and work ability on sickness absence among Dutch construction workers. Scand J Work Environ Health 2009;35:325-33.

14 Labriola M, Lund T, Burr H. Prospective study of physical and psychosocial risk factors for sickness absence. Occup Med 2006;56:469-74.

15 Ropponen A, Narusyte J, Alexanderson K, et al. Stability and change in health behaviours as predictors for disability pension: a prospective cohort study of Swedish twins. BMC Public Health $2011 ; 11$.

16 Beemsterboer W, Stewart R, Groothoff J, et al. A literature review on sick leave determinants (1984-2004). Int J Occup Med Environ Health 2009;22:169-79.

17 Floderus B, Göransson S, Alexanderson K, et al. Self-estimated life situation in patients on long-term sick leave. J Rehabil Med 2005;37:291-9.

18 Hogsbro C, Davidsen M, Sorensen J. Long-term sickness absence from work due to physical inactivity: a registry-based study. Scand J Public Health 2018;1403494817751328.

19 Lahti J, Laaksonen M, Lahelma E, et al. The impact of physical activity on sickness absence. Scand J Med Sci Sports 2010;20:191-9.

20 Robroek SJW, Schuring M, Croezen S, et al. Poor health, unhealthy behaviors, and unfavorable work characteristics influence pathways of exit from paid employment among older workers in Europe: a four year follow-up study. Scand J Work Environ Health 2013;39:125-33.

21 Fimland MS, Vie G, Johnsen R, et al. Leisure-time physical activity and disability pension: 9 years follow-up of the HUNT study, Norway. Scand J Med Sci Sports 2015;25:e558-65.

22 Amlani NM, Munir F. Does physical activity have an impact on sickness absence? A review. Sports Medicine 2014;44:887-907.

23 Holtermann A, Hansen JV, Burr $\mathrm{H}$, et al. The health paradox of occupational and leisure-time physical activity. $\mathrm{Br} J$ Sports Med 2012;46:291-5.

24 Sundstrup E, Hansen Ã...se Marie, Mortensen EL, et al. Cumulative occupational mechanical exposures during working life and risk of sickness absence and disability pension: prospective cohort study. Scand J Work Environ Health 2017;43:415-25.

25 Fine LJ, Philogene GS, Gramling R, et al. Prevalence of multiple chronic disease risk factors-2001 National Health Interview Survey. Am J Prev Med 2004;27:18-24.

26 Grant BF, Hasin DS, Chou SP, et al. Nicotine dependence and psychiatric disorders in the United States: results from the National Epidemiologic Survey on Alcohol and Related conditions. Arch Gen Psychiatry 2004;61:1107-15.

27 Piirtola M, Kaprio J, Silventoinen K, et al. Association between longterm smoking and leisure-time physical inactivity: a cohort study among Finnish twins with a 35-year follow-up. Int J Public Health 2017;62:819-29.

28 Ropponen A, Svedberg P. Single and additive effects of health behaviours on the risk for disability pensions among Swedish twins. Eur J Public Health 2014;24:643-8.

29 Noble N, Paul C, Turon $\mathrm{H}$, et al. Which modifiable health risk behaviours are related? A systematic review of the clustering of Smoking, Nutrition, Alcohol and Physical activity ('SNAP') health risk factors. Prev Med 2015;81:16-41.

30 Spring B, Moller AC, Coons MJ. Multiple health behaviours: overview and implications. J Public Health 2012;34:i3-10. 
31 Lichtenstein P, Sullivan PF, Cnattingius S, et al. The Swedish twin registry in the third millennium: an update. Twin Res Hum Genet 2006;9:875-82.

32 Ludvigsson JF, Svedberg P, Olén O, et al. The longitudinal integrated database for health insurance and labour market studies (LISA) and its use in medical research. Eur J Epidemiol 2019;34:423-37.

33 Wood AM, Kaptoge S, Butterworth AS, et al. Risk thresholds for alcohol consumption: combined analysis of individual-participant data for 599912 current drinkers in 83 prospective studies. The Lancet 2018;391:1513-23.

34 Trolle-Lagerros Y, Mucci LA, Kumle M, et al. Physical activity as a determinant of mortality in women. Epidemiology 2005;16:780-5.

35 Espman E, Allebeck P. Riskbruk av alkohol-begrepp, gränsvärden, mättmetoder [Risk use of alcohol-concepts, limit values, measurement methods. Stockholm: Karolinska Institutets folkhälsoakademi, 2011.

36 Mammen G, Faulkner G. Physical activity and the prevention of depression: a systematic review of prospective studies. Am J Prev Med 2013;45:649-57.

37 Powell KE, Paluch AE, Blair SN. Physical activity for health: what kind? how much? how intense? on top of what? Annu Rev Public Health 2011;32:349-65

38 Robroek SJW, Reeuwijk KG, Hillier FC, et al. The contribution of overweight, obesity, and lack of physical activity to exit from paid employment: a meta-analysis. Scand J Work Environ Health 2013;39:233-40.

39 Ekelund U, Ward HA, Norat T, et al. Physical activity and allcause mortality across levels of overall and abdominal adiposity in European men and women: the European prospective investigation into cancer and nutrition study (EPIC). Am J Clin Nutr 2015;101:613-21.
40 Jørgensen MB, Thygesen LC, Becker U, et al. Alcohol consumption and risk of unemployment, sickness absence and disability pension in Denmark: a prospective cohort study. Addiction 2017;112:1754-64.

41 Böckerman P, Hyytinen A, Maczulskij T. Devil in disguise: does drinking lead to a disability pension? Prev Med 2016;86:130-5.

42 Thern E, de Munter J, Hemmingsson T, et al. Effects of increased alcohol availability during adolescence on the risk of all-cause and cause-specific disability pension: a natural experiment. Addiction 2017;112:1004-12.

43 Schneider S, Huy C, Schuessler M, et al. Optimising lifestyle interventions: identification of health behaviour patterns by cluster analysis in a German 50+ survey. Eur J Public Health 2009;19:271-7.

44 Svedberg P, Ropponen A, Alexanderson K, et al. Genetic susceptibility to sickness absence is similar among women and men: findings from a Swedish twin cohort. Twin Res Hum Genet 2012;15:642-8.

45 Narusyte J, Ropponen A, Silventoinen K, et al. Genetic liability to disability pension in women and men: a prospective populationbased twin study. PLoS One 2011;6:e23143.

46 Althubaiti A. Information bias in health research: definition, pitfalls, and adjustment methods. J Multidiscip Healthc 2016;9:211-7.

47 Tucker JM, Welk GJ, Beyler NK. Physical activity in U.S.: adults compliance with the physical activity guidelines for Americans. Am J Prev Med 2011;40:454-61.

48 Troiano RP, Berrigan D, Dodd KW, et al. Physical activity in the United States measured by accelerometer. Med Sci Sports Exerc 2008;40:181-8.

49 Adams SAet al. The effect of social desirability and social approval on self-reports of physical activity. Am J Epidemiol 2005;161:389-98. 